\title{
Função sexual e qualidade de vida de mulheres: um estudo observacional
}

Larissa Santana Correia, ${ }^{1}$ Cristina Brasil, ${ }^{2}$ Marianne Dantas da Silva, ${ }^{1}$ Daiane Fernandes da Cunha Silva, ${ }^{1}$ Hortênsia Oliveira Amorim, ${ }^{1}$ Patrícia Lordêlo ${ }^{3}$

\section{RESUMO}

Objetivo: Apresentar a prevalência de disfunção sexual e comparar a relação entre função sexual e qualidade de vida em mulheres matriculadas em ginásios.

Tipo de estudo: Observacional transversal.

Local: Bahia - Brasil.

População: Mulheres matriculadas em ginásios.

Métodos: Trata-se de um estudo transversal, realizado em ginásios. Foram incluídas mulheres com idade entre 18 e 60 anos, sexualmente ativas e não grávidas. Foi utilizado um questionário com informações sociodemográficas e clínicas, além do Female Sexual Function Index que avaliou a função sexual, sendo considerados valores do score $\leq 26$ como disfunção sexual, enquanto a qualidade de vida foi avaliada pelo questionário SF-36. Na comparação dos valores médios encontrados nos scores do SF-36 entre as mulheres com disfunção sexual e adequada função sexual foi utilizado o teste $t$ de Student.

Resultados: A amostra foi constituída por 375 mulheres que praticam atividade física, com média de idade de 34,6 $\pm 10,0$ d.p. A frequência de disfunção sexual (DS) foi de $21 \%$ e, em todos os domínios do SF-36, as mulheres com DS tinham scores inferiores $(p<=0,01$ para os aspectos emocionais e $p<0,01$ para os outros domínios. Observaram-se diferenças mais expressivas nos domínios: saúde mental, aspectos físicos, aspectos emocionais e aspectos sociais, com média \pm desvio-padrão, respetivamente, 59,6 $618,7,72,6 \pm 33,1,67,1 \pm 38,7$ e $69,2 \pm 22,8$ para mulheres com DS e médias de $72,1 \pm 16,8,85,1 \pm 26,6,78,6 \pm 34,4$ e $80,7 \pm 21,9$ para mulheres com AFS ( $p<0,01$ em todos). Conclusão: A disfunção sexual nesta população tem impacto negativo na qualidade de vida.

Palavras-chave: Disfunção Sexual; Qualidade de Vida; Mulheres.

\section{INTRODUÇÃO}

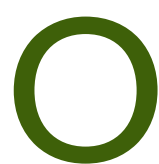
ciclo de resposta sexual divide-se em quatro frases: desejo, excitação, orgasmo e resolução. Cada fase apresenta uma característica e qualquer comprometimento em um dos estágios é definido como disfunção sexual (DS), ${ }^{1}$ considerando-se toda a situação em que a relação sexual não seja concretizada ou que esta seja insatisfatória para a mulher e para o parceiro. ${ }^{2}$

No Estudo do Comportamento Sexual foi detectado que $30 \%$ das mulheres referiam algum tipo de disfunção sexual. ${ }^{3}$ Num estudo realizado em mulheres portuguesas, $77 \%$ apresentavam disfunção sexual embora quase metade não a considerasse um problema. ${ }^{2}$ Outra pesquisa também descobriu que as mulheres com disfunção sexual apresentaram mais

'Fisioterapeuta pela Escola Bahiana de Medicina e Saúde Pública (EBMSP), Centro de Atenção ao Assoalho Pélvico da Escola Bahiana de Medicina e Saúde Pública ${ }^{2}$ Fisioterapeuta, Mestranda em Medicina e Saúde Humana pela Escola Bahiana de Medicina e Saúde Pública (EBMSP), Centro de Atenção ao Assoalho Pélvico da Escola Bahiana de Medicina e Saúde Pública

${ }^{3}$ Fisioterapeuta, Pós- doutora em Ginecologia pela UNIFESP, Doutora em Medicina e Saúde Humana, Docente dos programas de Pós-graduação de Medicina e Saúde Humana e Tecnologia em Saúde da EBMSP ansiedade e que a depressão está intimamente associada a disfunção sexual, com aumento do risco para o desenvolvimento da mesma. ${ }^{4}$

Embora frequente, muitas mulheres privam-se de tratamentos adequados por vergonha ou frustração ${ }^{5}$ ou por acharem que a disfunção sexual é intrínseca ao envelhecimento. A sexualidade da mulher com o avanço da idade é carregada de preconceito, neste período ainda existe o tabu da mulher assexuada. ${ }^{6}$ Apesar da alta prevalência de disfunção sexual, poucos centros no mundo realizam investigação abrangente e multidisciplinar e tratamento da disfunção sexual ou encaminhamento adequado visando a recuperação de uma adequada função sexual (AFS). As mulheres que praticam atividade física buscam procedimento cirúrgico devido a insatisfação com a região genital, por exemplo, como o uso de roupas justas que demarcam a vulva além do incómodo ocasionado pelo atrito e esta insatisfação pode estar relacionada com disfunções sexuais..$^{7-8}$

A literatura brasileira ainda se mostra escassa na avaliação do real impacto que as disfunções sexuais podem provocar na qualidade de vida (QV) da mulher e com a utilização de instrumentos de avaliação validados para realizar tal análise. ${ }^{9}$ Estudos de incidência e prevalência mostram-se deficientes quan- 
to a metodologia e tamanho da amostra. ${ }^{10}$ Além disso, verifica-se escassez na análise da influência na qualidade de vida em mulheres que praticam atividadefísica. Tendo em vista os transtornos que as disfunções sexuais podem causar e os benefícios que poderá proporcionar o melhor entendimento da relação da DS coma QV no público feminino, o objetivo deste estudoé descrever a frequência e comparar a relação entre função sexual e qualidade de vida em mulheres praticantes de atividade física.

\section{MÉTODOS}

Trata-se de um estudo observacional, de corte transversal, tendo a colheita de dados sido realizada no período entre Fevereiro e Junho de 2014, entre as mulheres matriculadas em três grandes ginásios, localizados na cidade de Salvador e Lauro de Freitas-BA. Foram incluídas mulheres na faixa etária entre 18 e 60 anos, sexualmente ativas nas últimas quatro semanas e não grávidas, matriculadas em ginásios. Foram excluídas da análise aquelas que não preencheram os instrumentos de avaliação propostos na íntegra.

As mulheres foram convidadas pelas pesquisadoras a participar no estudo, pessoalmente, nos ginásios. As que manifestaram interesse foram direcionadas para preencher os questionários autoaplicáveis num local reservado - informações sociodemográficas como: idade, grau de escolaridade, estado civil, rendimento; além de dados clínicos: IMC, história obstétrica e ginecológica, uso de medicamentos e número de cirurgias pélvicas/perineais integravam os questionários.

A função sexual foi avaliada por meio do FSFI (Female Sexual Function Index), um questionário traduzido e validado para a língua portuguesa. ${ }^{8}$ Constituído por 19 questões e seis domínios (desejo, excitação sexual, lubrificação vaginal, orgasmo, satisfação sexual e dor), com uma pontuação total que pode variar de 2 a 36 pontos, onde valores $\leq 26$ indicam disfunção sexual.

Foi utilizado o questionário multidimensional SF-36 (Medical Outcomes Study 36-Item Short-Form Health Survey) para avaliar a qualidade de vida. Composto por 36 itens, reunidos em duas componentes: física e mental que avaliam a capacidade funcional (desempenho das atividades diárias, como capacidade de cuidar de si, vestir-se, tomar banho e subir escadas); aspectos físicos (impacto da saúde física no desempenho das atividades diárias e/ou profissionais); dor (nível de dor e o impacto no desempenho das atividades diárias e ou profissionais); estado geral de saúde (percepção do estado geral de saúde); vitalidade (percepção do estado de saúde); aspectos sociais (reflexo da condição de saúde física nas atividades sociais); aspectos emocionais (reflexo das condições emocionais no desempenho das atividades diárias e/ou profissionais); e saúde mental (escala de humor e bemestar). A pontuação final de cada domínio varia de 0 a 100, não existindo um ponto de corte definido: valores de scores maiores indicam um melhor estado de saúde. ${ }^{9}$

O cálculo amostral foi realizado pela calculadora Winpepi (http://www.brixtonhealth.com/pepi4windows.html) para uma estimativa de proporção, usando como desfecho principal a função sexual. Foi utilizada uma proporção de $30 \%$, que representa a prevalência de mulheres com disfunção sexual no Brasil, ${ }^{11}$ com uma diferença aceitável de \pm 5 , nível de confiança de $95 \%$, totalizando 323 participantes; acrescido $10 \%$ para possíveis perdas, seriam então necessárias 355 mulheres.

Para elaboração do banco de dados, análise descritiva e análise estatística foi utilizado o software Statistical Package for Social Sciences (SPSS), versão 14.0 para Windows. A normalidade das variáveis numéricas foi verificada através da estatística descritiva, análise gráfica e do teste Kolmogorov Sminorv. Os dados categóricos (estado civil, escolaridade, renda, IMC, tipo de parto, uso de medicamentos, menopausa, cirurgia pélvica/perineal e scores do FSFI) foram apresentados em frequência absoluta, enquanto os dados numéricos (idade, scores dos domínios do SF-36) foram apresentados em termos de média e desvio-padrão. Para a comparação das médias dos scores do SF-36 das mulheres com disfunção sexual e das mulheres com adequada função sexual foi utilizado o teste $t$ de Student, pressupondo uma distribuição normal. Como referido, para a comparação das variáveis numéricas com a disfunção sexual foi utilizado o teste $t$ de Student e, para a comparação das variáveis categóricas com a disfunção sexual, o qui-quadrado.

Este estudo faz parte de um projeto que foi aprovado pelo Comitê de Ética e Pesquisa (CEP) da Escola Bahiana de Medicina e Saúde Pública (EBMSP), CAAE n. ${ }^{\circ}$ 14425813.9.0000.5544, conforme rege a resolução n. ${ }^{\circ} 466 / 12$ do Conselho Nacional de saúde. Todas as participantes assinaram o termo de consentimento livre e esclarecido (TCLE).

\section{RESULTADOS}

Das 385 mulheres investigadas, 10 foram excluídas da análise pelo preenchimento incompleto dos instrumentos de avaliação, resultando numa amostra de 375 mulheres. No Quadro I constata-se uma predominância de mulheres jovens, com menos de 40 anos, com idade mínima de 18 e máxima de 57 anos. A maioria das mulheres eram casadas (49,3\%), apresentaram o ensino superior completo $(73,6 \%)$. Quanto às características clínicas, a maioria das mulheres apresentaram IMC eutrófico (69,2\%), não tiveram nenhum parto (55,9\%), faziam uso de anticoncepcional oral $(71,9 \%)$, estavam em idade fértil (89\%) e não realizaram cirurgia ginecológica (84,5\%).

Na comparação dos dados sociodemográficos e clínicos entre as mulheres com adequada função sexual (AFS) e as com disfunção sexual (DS) pode-se perceber uma maior 


\begin{tabular}{|c|c|c|c|c|}
\hline \multicolumn{5}{|c|}{$\begin{array}{l}\text { QUADRO I. Comparação das características sociodemográficas e clínicas } \\
\text { das mulheres sexualmente ativas (AFS e DS) matriculadas em ginásios da } \\
\text { cidade de Salvador e Lauro de Freitas (BA), } 2014\end{array}$} \\
\hline Variáveis & $\begin{array}{c}\text { TOTAL } \\
\text { Média } \pm \text { DP }\end{array}$ & $\begin{array}{c}\text { AFS } \\
(n=296)\end{array}$ & $\begin{array}{c}\text { DS } \\
(n=79)\end{array}$ & $P$-valor \\
\hline Idade & $34,6 \pm 10,0$ & $34,2 \pm 9,6$ & $36,0 \pm 10,9$ & 0,154 \\
\hline Estado civil & $n(\%)$ & & & \\
\hline Casadas & $184(49,3)$ & $142(48,1)$ & $42(53,8)$ & 0,471 \\
\hline Solteiras & $178(47,7)$ & $143(48,5)$ & $35(44,9)$ & \\
\hline Divorciadas & $11(2,9)$ & $10(3,4)$ & $1(1,3)$ & \\
\hline \multicolumn{5}{|l|}{ Escolaridade } \\
\hline Ensino superior completo & $276(73,6)$ & $223(75,3)$ & $53(67,1)$ & 0,113 \\
\hline Ensino superior incompleto & $67(17,9)$ & $53(17,9)$ & $14(17,7)$ & \\
\hline Ensino médio & $30(8)$ & $19(6,4)$ & $11(13,9)$ & \\
\hline Ensino fundamental & $2(0,5)$ & $1(0,3)$ & $1(1,3)$ & \\
\hline \multicolumn{5}{|l|}{ IMC } \\
\hline Eutróficos & $249(69,2)$ & $200(69,9)$ & $49(66,2)$ & $0,025^{*}$ \\
\hline Sobrepeso & $85(23,6)$ & $71(24,8)$ & $14(18,9)$ & \\
\hline Obesidade & $22(6,1)$ & $12(4,2)$ & $10(13,5)$ & \\
\hline Abaixo do peso & $4(1,1)$ & $3(1,0)$ & $1(1,4)$ & \\
\hline \multicolumn{5}{|l|}{ Tipo de partos } \\
\hline Nenhum parto & $207(55,9)$ & $168(57,1)$ & $39(51,3)$ & 0,140 \\
\hline Cesariana & $120(32,4)$ & $89(30,3)$ & $31(40,8)$ & \\
\hline Normal & $43(11,6)$ & $37(12,6)$ & $6(7,9)$ & \\
\hline \multicolumn{5}{|l|}{ Medicamentos } \\
\hline Uso de hormonas & $228(71,9)$ & $182(72,2)$ & $46(70,8)$ & 0,816 \\
\hline \multicolumn{5}{|l|}{ Fase hormonal } \\
\hline Idade fértil & 323 (89) & $261(91,3)$ & $62(80,5)$ & $0,008^{*}$ \\
\hline \multicolumn{5}{|l|}{ Cirurgia ginecológica } \\
\hline Ausente & $317(84,5)$ & $250(86,5)$ & $67(85,9)$ & 0,890 \\
\hline
\end{tabular}

Legenda: $n=$ número de participantes; IMC=Índice de massa corporal; AFS=Adequada função sexual; DS=Disfunção sexual; *Teste qui-quadrado.

presença de mulheres obesas $[10(13,5)]$ no grupo de mulheres com DF quando comparadas com $12(4,2)$ mulheres com AFS $(p=0,025)$. Outra diferença encontrada foi a presença de uma maior proporção de mulheres na idade fértil no grupo com AFS $[261(91,3)]$ quando comparado com 62 $(80,5)$ no grupo com DS ( $p=0,008)$ (Quadro I).

Foi observada uma frequência de $21,1 \%$ de mulheres com DS, o que representa 79 mulheres. No Quadro II foram comparadas as médias dos domínios do questionário de qualidade de vida SF-36 nas mulheres com AFS e com DS, sendo verificado que as médias das pontuações das mulheres com
DS se apresentaram menores em todos os domínios analisados, com significância estatística.

\section{DISCUSSÃO}

O presente estudo avaliou a frequência de disfunção sexual e a relação com a qualidade de vida em mulheres matriculadas em ginásios e demonstrou uma frequência de $21 \%$ de DS, com uma associação com menores valores em todos os domínios de qualidade de vida. Foi comparada a qualidade de vida entre as mulheres com disfunção sexual e com adequada função sexual e os achados se mostraram semelhantes aos encontrados na literatura. ${ }^{11-12}$ Este resultado se deu principalmente pela utilização de questionários validados para a língua portuguesa, amplamente utilizados na literatura e designados especificamente para a análise da função sexual e da qualidade de vida.

Na avaliação da qualidade de vida em todos os domínios do SF-36, as mulheres com DS apresentaram pontuações em média inferiores, principalmente nos domínios de saúde mental, aspectos físicos, aspectos emocionais e aspectos sociais. Estes dados sugerem que mulheres com DS apresentam um maior comprometimento da qualidade de vida nestes quatro aspectos avaliados. Esses domínios estão relacionados com questões físicas e mentais, ressaltando o comprometimento em ambos os quesitos. Este achado corrobora os resultados de um estudo realizado com mulheres americanas, onde foi destacado que mulheres com disfunção sexual apresentam insatisfação física e emocional, com sentimentos de infelicidade. ${ }^{11} \mathrm{O}$ resultado do presente estudo ratifica a literatura existente, uma vez que o bem-estar geral está associado a uma saúde sexual satisfatória, ${ }^{13}$ sendo um fator importante na qualidade de vida e considerada fundamental, mesmo em mulheres praticantes de atividade física. ${ }^{14}$

A frequência de disfunção sexual neste estudo com mulheres praticantes de atividade física é semelhante a alguns estudos realizados no Brasil sobre a disfunção sexual em mulheres; ${ }^{3,15}$ semelhante ao percentual de DS (21,9\%) encontrado por Prado e seus colaboradores (2010) ao analisarem mulheres, com a idade compreendida entre os 18 e os 45 anos, com diferentes níveis sociais socioeconômicos. ${ }^{15} \mathrm{~A}$ equivalência na frequência 


\begin{tabular}{|c|c|c|c|}
\hline \multicolumn{4}{|c|}{$\begin{array}{l}\text { QUADRO II. Comparação dos scores dos domínios do } \\
\text { questionário SF-36 das mulheres com adequada função } \\
\text { sexual e disfunção sexual, sexualmente ativas, } \\
\text { matriculadas em ginásios da cidade de Salvador e Lauro } \\
\text { de Freitas (BA), } 2014\end{array}$} \\
\hline Domínios SF-36 & AFS & DS & $\mathbf{P}$ \\
\hline Capacidade funcional & $90,3 \pm 13,0$ & $83,8 \pm 15,5$ & $<0,01$ \\
\hline Aspectos físicos & $85,1 \pm 26,6$ & $72,6 \pm 33,1$ & $<0,01$ \\
\hline Dor & $73,9 \pm 20,2$ & $66,5 \pm 20,8$ & $<0,01$ \\
\hline Estado geral & $79,9 \pm 16,4$ & $72,7 \pm 18,5$ & $<0,01$ \\
\hline Vitalidade & $63,6 \pm 18,1$ & $56,2 \pm 19,4$ & $<0,01$ \\
\hline Aspectos sociais & $80,7 \pm 21,9$ & $69,2 \pm 22,8$ & $<0,01$ \\
\hline Aspectos emocionais & $78,6 \pm 34,4$ & $67,1 \pm 38,7$ & 0,01 \\
\hline Saúde mental & $72,1 \pm 16,8$ & $59,6 \pm 18,7$ & $<0,01$ \\
\hline
\end{tabular}

Legenda: Média $\pm D P$ - Teste $t$ de Student; AFS=Adequada função sexual; DS=Disfunção sexual.

pode ser explicada pela semelhança das idades das populações estudadas, uma vez que num estudo com mulheres de meia idade (40 a 65 anos), na cidade de Natal, foi referido $67 \%$ de DS.

No entanto, a prevalência de disfunção sexual ainda se mostra divergente quanto a literatura no âmbito mundial. Estudos mostram uma variação de prevalência entre $22 \%$ e 87\% em diferentes populações femininas..$^{2,11,16-18}$ A grande variação é, em parte, devida à dimensão e características de amostras, tipos de testes realizados e tipos de questionários utilizados, que podem carecer de uma precisão das respostas. ${ }^{11}$ West e seus colaboradores afirmaram que a dificuldade de resultados precisos sobre a DS parece ser pela falta de dados sobre a população em geral, uma vez que encontrar uma grande amostra representativa é desafiador e caro, ${ }^{19}$ além das questões culturais ${ }^{9}$ e religiosas e da falta de conhecimento do próprio corpo e de uma ou mais fases do ciclo da resposta sexual, que podem interferir na função sexual e, consequentemente, na frequência encontrada. ${ }^{20}$

$\mathrm{Na}$ amostra estudada houve um predomínio de mulheres jovens, com menos de 40 anos e que estavam em idade fértil e este é um fator a ser considerado quanto à frequência de DS encontrada, pois mulheres mais jovens tendem a ter menos comprometimentos sexuais. A literatura mostra que, na menopausa, o corpo da mulher sofre modificações pela queda do estrogênio, o que favorece o surgimento de alterações nas fases da resposta sexual. A diminuição do desejo sexual, assim como diminuição da lubrificação vaginal e dispareunia são frequentemente relatados por essas mulheres. ${ }^{7} \mathrm{Um}$ estudo com brasileiras mostrou que, a partir dos 50 anos de idade, a disfunção sexual aumenta quando comparada com as mulheres com menos de 50 anos de idade. ${ }^{21}$ Dennerstein, num estudo com mulheres australianas, conseguiu demonstrar que a função sexual declina com a menopausa e que o estradiol tem uma relação significativa com este declínio. ${ }^{22}$

Outra importante consideração quanto à frequência encontrada é o fato da população analisada ser representada por mulheres que praticam atividade física, uma vez que a literatura tem demonstrado um efeito indireto, porém positivo, da prática de atividade física sobre a função sexual através da produção de hormonas. Um estudo com brasileiras demonstrou, a partir do FSFI, uma maior prevalência de disfunção sexual em mulheres menos ativas ou sedentárias quando comparadas a outras que praticavam atividade física regular, demonstrando que mulheres que praticam atividade física apresentam uma melhor função sexual. ${ }^{16}$

Um ponto limitante deste estudo é a impossibilidade de comparar os dados com a população feminina em geral, uma vez que o presente estudo avaliou apenas mulheres que praticam atividade física. Independentemente disso, concluiu-se que mulheres praticantes de atividade física têm uma frequência de $21 \%$ de disfunção sexual e verificou-se associação desta com scores na qualidade de vida (SF-36) inferiores, principalmente nos aspectos físicos, emocionais e sociais, interferindo no bem-estar. Contudo, a validade da associação entre disfunção sexual e qualidade de vida deve ser lida com precaução, dada a natureza observacional do estudo à eventual presença de viés de confundimento.

Este trabalho alerta para a importância da adequada função sexual na qualidade de vida global das mulheres, mesmo aquelas que são fisicamente ativas. Pesquisas demonstram a importância de considerar as diferenças individuais ao falar sobre as disfunções sexuais, que vão além da componente física e hormonal. O sentimento de satisfação com a vida sexual está intrinsecamente relacionado com as experiências sexuais passadas do indivíduo, expectativas atuais e aspirações futuras, ${ }^{23}$ sendo também presente uma alta associação entre a disfunção sexual com experiências e relações pessoais insatisfatórias. ${ }^{3}$ Considerando a importância da saúde sexual para o bem-estar completo, a investigação futura dever-se-á focar em avaliar a associação da DS com a QV na população feminina em geral. Além disso, serão necessários estudos longitudinais que avaliem a relação bidirecional da função sexual e da qualidade de vida em mulheres.

\section{REFERÊNCIAS BIBLIOGRÁFICAS}

1. Pereira VM, Silva AC, Nardi AE. Transtorno da excitação genital persistente: uma revisão da literatura [Persistent genital arousal disorder: a literature review]. J Bras Psiquiatr. 2010;59(3):223-32. Portuguese

2. Ribeiro B, Magalhães AT, Mota I. Disfunção sexual feminina em idade reprodutiva prevalência e fatores associados [Female sexual dysfunction in the reproductive years: prevalence and associated factors]. Rev Port Med Geral Fam. 2013;29(1):16-24. Portuguese 
3. Abdo $\mathrm{CH}$, Oliveira JrWM, Moreira Junior ED, Fittipaldi JA. Perfil sexual da população brasileira: resultados do Estudo do Comportamento Sexual (ECOS) do brasileiro [Sexual profile of brazilian population: results from Brazilian Study of Sexual Behavior (BSSB)]. Rev Bras Med. 2002;59(4):250-7. Portuguese

4. Pereira VM, Nardi AE, Silva AC. Sexual dysfunction, depression, and anxiety in young women according to relationship status: an online survey. Trends Psychiatry Psychother. 2013;35(1):55-61.

5. Ferreira CC, Mota LM, Oliveira AC, Carvalho JF, Lima RA, Simaan CK, et al. Frequência de disfunção sexual em mulheres com doenças reumáticas [Frequency of sexual dysfunction in women with rheumatic diseases]. Rev Bras Reumatol. 2013;53(1):416. Portuguese

6. Secretaria de Atenção à Saúde. Manual de atenção à mulher no climatério / menopausa [Internet]. Brasília, DF: Ministério da Saúde; 2008. ISBN 9788533414860. Available from: http://bibliofarma.com/manual-de-atencao-a-mulher-no-climateriomenopausa/

7. Pasqualotto EB, Pasqualotto FF, Sobreiro BP, Lucon AM. Female sexual dysfunction: the important points to remember. Clinics. 2005;60(1):51-60.

8. Crouch NS, Deans R, Michala L, Liao LM, Creighton SM. Clinical characteristics of well women seeking labial reduction surgery: a prospective study. BJOG. 2011;118(12):1507-10.

9. Thiel RR, Dambros M, Palma PC, Thiel M, Riccetto CL, Ramos MF. Tradução para português, adaptação cultural e validação do Female Sexual Function Index [Translation into Portuguese, cross-national adaptation and validation of the Female Sexual Function Index]. Rev Bras Ginecol Obstet. 2008;30(10):504-10. Portuguese

10. Gabbard GO. Musings on the report of the International Consensus Development Conference on Female Sexual Dysfunction: definitions and classifications. J Sex Marital Ther. 2001;27(2):145-7

11. Cohen PG. Sexual dysfunction in the United States. JAMA. 1999;282(13):1229.

12. Atlantis E, Sullivan T: Bidirectional association between depression and sexual dysfunction: a systematic review and meta-analysis. J Sex Med. 2012;9(6):1497-1507.

13. Mulhall J, King R, Glina S, Hvidsten K. Importance of and satisfaction with sex among men and women worldwide: results of the global better sex survey. J Sex Med. 2008;5(4):788-95.

14. Feldhaus-Dahir M. Female sexual dysfunction: barriers to treatment. Urol Nurs. 2009;29(2):81-5

15. Prado DS, Mota VP, Lima TI. Prevalência de disfunção sexual em dois grupos de mulheres de diferentes níveis socioeconômicos [Prevalence of sexual dysfunction in two women groups of different socioeconomic status]. Rev Bras Ginecol Obstet.
2010;32(3):139-43. Portuguese

16. Cabral PU, Canário AC, Spyrides MH, Uchôa SA, Eleutério Júnior J, Giraldo PC, et al. Physical activity and sexual function in middle-aged women. Rev Assoc Med Bras. 2014;60(1):47-52.

17. Jaafarpour M, Khani A, Khajavikhan J, Suhrabi Z. Female sexual dysfunction: prevalence and risk factors. J Clin Diagn Res. 2013;7(12):2877-80.

18. Jamali $S$, Zarei $H$, Jahromi AR. The relationship between body mass index and sexual function in infertile women: a cross-sectional survey. Iran J Reprod Med. 2014; 12(3):189-98.

19. West SL, Vinikoor LC, Zolnoun D.A systematic review of the literature on female sexual dysfunction prevalence and predictors. Annu Rev Sex Res. 2004;15:40-172.

20. Briedite I, Ancane G, Ancans A, Erts R. Insufficient assessment of sexual dysfunction: a problem in gynecological practice. Medicina (Kaunas). 2013;49(7):315-20.

21. Valadares AL, Pinto-Neto AM, Osis MJ, Conde DM, Sousa MH, Costa-Paiva L. Sexuality in Brazilian women aged 40 to 65 years with 11 years or more of formal education: associated factors. Menopause. 2008;15(2):264-9.

22. Dennerstein L, Randolph J, Taffe J, Dudley E, Burger H. Hormones, mood, sexuality, and the menopausal transition. Fertil Steril. 2002;77 Suppl 4:S42-8.

23. Davidson J, Darling C, Norton L. Religiosity and the sexuality of women: sexual behaviour and sexual satisfaction revisited. J Sex Res. 1995;32(3):235-43.

\section{CONFLITO DE INTERESSES}

As autoras declaram não ter conflitos de interesses.

\section{COMISSÃO DE ÉTICA}

Estudo realizado após parecer favorável do Comité de Ética em Pesquisa da Escola Bahiana de Medicina e Saúde Pública/Brasil.

\section{ENDEREÇO PARA CORRESPONDÊNCIA}

Patrícia Lordêlo

Escola Bahiana de Medicina e Saúde Pública, Av. Dom João VI, n 275, Brotas, Salvador - Bahia, CEP 40290-00

E-mail: pvslordelo@hotmail.com

Recebido em 29-07-2015

Aceite para publicação em 01-11-2016

Artigo redigido em Português do Brasil.

\section{ABSTRACT}

\section{SEXUAL FUNCTION AND QUALITY OF LIFE IN WOMEN: A CROSS SECTIONAL STUDY}

Objective: To compare the relationship between sexual function and quality of life (QOL) in women.

Type of study: Cross-sectional study.

Setting: Bahia, Brazil

Participants: High school educated women who were members of a fitness club.

Methods: Sexually active non-pregnant women between 18 and 60 years of age were invited to participate in this study. A questionnaire on sociodemographic and clinical information was administered along with the Female Sexual Function Index. Quality of life was evaluated using the SF-36 questionnaire. SF-36 scores of women with and without sexual were compared using the independent $t$ test.

Results: The sample included 375 physically active women with a mean age of $34.6 \pm 10.0$ years. Sexual dysfunction was found in $21.1 \%$. In all domains of the SF-36 women with dysfunction had lower mean scores ( $p \leq 0.01$ for emotional aspects and $p<0.01$ for other domains). We observed differences in mean scores in mental health, physical, emotional aspects and social aspects respectively, (59.6 \pm 18.7$)$, $(72.6 \pm 33.1),(67.1 \pm 38.7)$ and $(69.2 \pm 22.8)$ for women with sexual dysfunction and in the median scores $(72.1 \pm 16.8),(85.1 \pm 26.6)$, $(78.6 \pm 34.4)$ and $(80.7 \pm 21.9)$ for women with adequate sexual function $(p<0,01$ for all).

Conclusion: Sexual Dysfunction had a negative impact on $\mathrm{QOL}$ in women in this population.

Keywords: Sexual Dysfunction; Quality of Life; Women; Physical Activity. 